\title{
THE COMPLEXITY OF TOPOLOGICAL GROUP ISOMORPHISM
}

\author{
ALEXANDER S. KECHRIS, ANDRÉ NIES AND KATRIN TENT
}

\begin{abstract}
We study the complexity of the isomorphism relation for various classes of closed subgroups of $S_{\infty}$. We use the setting of Borel reducibility between equivalence relations on Polish spaces. For profinite, locally compact, and Roelcke precompact groups, we show that the complexity is the same as the one of countable graph isomorphism. For oligomorphic groups, we merely establish this as an upper bound.
\end{abstract}

\section{INTRODUCTION}

Let $S_{\infty}$ denote the Polish group of permutations of $\omega$. It is well-known that the closed subgroups of $S_{\infty}$ (or equivalently, the non-Archimedean Polish groups) are, up to topological group isomorphism, the automorphism groups of countable structures. Algebra or model theory can sometimes be used to understand natural classes of closed subgroups of $S_{\infty}$. Firstly, the separable profinite groups are precisely the Galois groups of Galois extensions of countable fields. For a second example, consider the oligomorphic groups, namely the closed subgroups of $S_{\infty}$ such that for each $n$ there are only finitely many $n$-orbits. They are precisely the automorphism groups of $\omega$-categorical structures. Under this correspondence, topological isomorphism turns into bi-interpretability of the structures by a result of Ahlbrandt and Ziegler [1] sometimes also attributed to Coquand.

The closed subgroups of $S_{\infty}$ form the points of a standard Polish space. Our main goal is to determine the complexity of the topological isomorphism relation for various classes of closed subgroups of $S_{\infty}$ within the setting of Borel reducibility between equivalence relations. See [4] for background on this setting. An important question about an equivalence relation $E$ on a standard Borel space $X$ is whether $E$ is classifiable by countable structures. This means that one can in a Borel way assign to $x \in X$ a countable structure $M_{x}$ in a fixed countable language so that $x E y \Leftrightarrow M_{x} \cong M_{y}$. We consider the Borel classes of compact (i.e., profinite) groups, locally compact groups, and oligomorphic groups. We also include the class of Roelcke precompact groups, which generalise both the compact and the oligomorphic groups.

We introduce a general criterion on a class of closed subgroups of $S_{\infty}$ to show that each of the classes above has an isomorphism relation that is classifiable by countable structures. Our proof that the criterion works has two different versions. The first is on the descriptive set theory side: we Borel reduce the isomorphism relation to conjugacy of closed subgroups of $S_{\infty}$, which implies classifiability by countable structures using a result

The first author was partially supported by NSF grant DMS 1464475. The second author was partially supported by the Marsden fund of New Zealand. The third author was supported by Sonderforschungsbereich 878 at Universität Münster. 
due to Becker and Kechris [2, Thm. 2.7.3]. The second is on the model theoretic side: we directly construct a countable structure $M_{G}$ in a fixed finite language from a group $G$ in the class so that topological isomorphism of groups is equivalent to isomorphism of the associated structures.

Independently from us, Rosendal and Zielinski [10, Prop. 10 and 11] established classifiability by countable structures for the isomorphism relation in the four classes above, and published their result on arXiv in Oct. 2016. Their methods are different from ours: they obtain the results as corollaries to their theory of classification by compact metric structures.

We conversely provide a Borel reduction of graph isomorphism to isomorphism of profinite groups, using an extension of an argument by Mekler [7] in the framework of topological groups. In fact, for $p$ an odd prime, the class of exponent $p$, nilpotent of class 2, profinite groups suffices.

For isomorphism of oligomorphic groups, it is clear that the identity on $\mathbb{R}$ is a lower bound (e.g. using Henson digraphs); we leave open the question whether this lower bound can be improved. We note that for $\omega$-categorical structures in a finite language, the bi-interpretability relation is Borel and has countable equivalence classes. Since graph isomorphism is not Borel, this upper bound for the isomorphism relation of the corresponding automorphism groups is not sharp.

Using Lemma 2.1 below, it is not hard to verify that the isomorphism relation for general closed subgroups of $S_{\infty}$ is analytical. It is unknown what the exact complexity of this relation is in terms of Borel reducibility. By the above, graph isomorphism is a lower bound.

\section{Preliminaries}

Effros structure of a Polish space. Given a Polish space $X$, let $\mathcal{F}(X)$ denote the set of closed subsets of $X$. The Effros structure on $X$ is the Borel space consisting of $\mathcal{F}(X)$ together with the $\sigma$-algebra generated by the sets

$$
\mathcal{C}_{U}=\{D \in \mathcal{F}(X): D \cap U \neq \emptyset\},
$$

for open $U \subseteq X$. Clearly it suffices to take all the sets $U$ in a countable basis $\left\langle U_{i}\right\rangle_{i \in \omega}$ of $X$. The inclusion relation on $\mathcal{F}(X)$ is Borel because for $C, D \in \mathcal{F}(X)$ we have $C \subseteq D \leftrightarrow \forall i \in \mathbb{N}\left[C \cap U_{i} \neq \emptyset \rightarrow D \cap U_{i} \neq \emptyset\right]$.

The following fact will be used frequently.

Lemma 2.1 (see [6], Thm. 12.13). Given a Polish space $X$, there is a Borel map $f: \mathcal{F}(X) \longrightarrow X^{\omega}$ such that for a non-empty set $G \in \mathcal{F}(X)$, the image $f(G)$ is a sequence $\left(p_{i}^{G}\right)_{i \in \omega}$ in $X^{\omega}$ that is dense in $G$.

The Effros structure of $S_{\infty}$. For a Polish group $G$, we have a Borel action $G \curvearrowright \mathcal{F}(G)$ given by left translation. In this paper we will only consider the case that $G=S_{\infty}$. In the following $\sigma, \tau, \rho$ denote injective maps on initial segments of the integers, that is, tuples of integers without repetitions. Let $[\sigma]$ denote the set of permutations extending $\sigma$ :

$$
[\sigma]=\left\{f \in S_{\infty}: \sigma \prec f\right\}
$$

(this is often denoted $\mathcal{N}_{\sigma}$ in the literature). The sets $[\sigma]$ form a base of $S_{\infty}$. For $f \in S_{\infty}$ let $f \uparrow_{n}$ be the initial segment of $f$ of length $n$. Note that the $\left[f\left\lceil_{n}\right]\right.$ form a basis of neighbourhoods of $f$. Given $\sigma, \sigma^{\prime}$ let $\sigma^{\prime} \circ \sigma$ be the composition as far as it is defined; for instance, $(7,4,3,1,0) \circ(3,4,6)=(1,0\rangle$. 
Definition 2.2. For $n \geq 0$, let $\tau_{n}$ denote the function $\tau$ defined on $\{0, \ldots, n\}$ such that $\tau(i)=i$ for each $i \leq n$.

Definition 2.3. For $P \in \mathcal{F}\left(S_{\infty}\right)$, by $T_{P}$ we denote the tree describing $P$ as a closed set in the sense that $\left[T_{P}\right] \cap S_{\infty}=P$. Note that $T_{P}=\left\{\sigma: P \in \mathcal{C}_{[\sigma]}\right\}$.

Lemma 2.4. The relation $\{(A, B, C): A B \subseteq C\}$ on $\mathcal{F}\left(S_{\infty}\right)$ is Borel.

Proof. $A B \subseteq C$ is equivalent to the Borel condition

$$
\forall \beta \in T_{B} \forall \alpha \in T_{A}\left[|\alpha|>\max \beta \rightarrow \alpha \circ \beta \in T_{C}\right] .
$$

For the nontrivial implication, suppose the condition holds. Given $f \in$ $A, g \in B$ and $n \in \mathbb{N}$, let $\beta=g \uparrow_{n}$, and $\alpha=f \uparrow_{1+\max \beta}$. Since $\alpha \circ \beta \in T_{C}$, the neighbourhood $\left[f \circ g\left\lceil_{n}\right]\right.$ intersects $C$. As $C$ is closed and $n$ was arbitrary, we conclude that $f \circ g \in C$.

\section{The Borel space of non-Archimedean groups.}

Lemma 2.5. The closed subgroups of $S_{\infty}$ form a Borel set $\mathcal{U}\left(S_{\infty}\right)$ in $\mathcal{F}\left(S_{\infty}\right)$.

Proof. $D \in \mathcal{F}\left(S_{\infty}\right)$ is a subgroup iff the following three conditions hold:

- $D \in \mathcal{C}_{[(0,1, \ldots, n-1\rangle]}$ for each $n$

- $D \in \mathcal{C}_{[\sigma]} \rightarrow D \in \mathcal{C}_{\left[\sigma^{-1}\right]}$ for all $\sigma$

- $D \in \mathcal{C}_{[\sigma]} \cap C_{[\tau]} \rightarrow D \in \mathcal{C}_{[\tau \circ \sigma]}$ for all $\sigma, \tau$.

It now suffices to observe that all three conditions are Borel.

Note that $\mathcal{U}\left(S_{\infty}\right)$ is a standard Borel space. The statement of the lemma actually holds for each Polish group in place of $S_{\infty}$.

Locally compact closed subgroups of $S_{\infty}$. These groups are exactly the (separable) totally disconnected locally compact groups. The class of such groups has been widely studied. A set $D \in \mathcal{F}\left(S_{\infty}\right)$ is compact iff the tree $T_{D}=\left\{\sigma: D \in \mathcal{C}_{[\sigma]}\right\}$ is finite at each level. A closed subgroup $G$ of $S_{\infty}$ is locally compact iff some point in $G$ has a compact neighbourhood. Equivalently, there is $\tau$ such that $G \in \mathcal{C}_{[\tau]}$ and the tree $\left\{\sigma \succeq \tau: G \in \mathcal{C}_{[\sigma]}\right\}$ is finite at each level. Thus, compactness and local compactness of subgroups are Borel conditions in $\mathcal{F}\left(S_{\infty}\right)$.

The canonical structure for a closed subgroup of $S_{\infty}$. Given $G \in$ $\mathcal{U}\left(S_{\infty}\right)$ we can in a Borel way obtain a countable structure $M_{G}$ in a countable signature such that $G \cong \operatorname{Aut}\left(M_{G}\right)$. For each $n$, order the $n$-tuples lexicographically. Let $\left\langle\bar{a}_{i}\right\rangle_{i<k_{n}}$, where $k_{n} \leq \omega$, be the ascending list of the $n$-tuples that are least in their orbits. The signature has $n$-ary predicate symbols $P_{i}^{n}$ for $i<k_{n}$, where the symbol $P_{i}^{n}$ is interpreted as the $G$-orbit of $\bar{a}_{i}$.

\section{A SUFFICIENT CRITERION FOR CLASSIFIABILITY OF TOPOLOGICAL ISOMORPHISM BY COUNTABLE STRUCTURES}

We show that the isomorphism relation on a Borel class $\mathcal{V}$ of subgroups of $S_{\infty}$ that is invariant under conjugacy is classifiable by countable structures, provided that we can canonically assign to each $G \in \mathcal{V}$ a countable base of neighbourhoods of 1 that are open subgroups. For instance, in the case of locally compact groups $G$, we can take as $\mathcal{N}_{G}$ the compact open subgroups 
of $G$. This relies on the classic result of van Dantzig: if a totally disconnected group $G$ is locally compact, it has a compact open subgroup $U$. Note that $U$ is profinite, so in fact 1 has a basis of neighbourhoods consisting of compact open subgroups.

\subsection{The sufficient criterion.}

Theorem 3.1. Let $\mathcal{V}$ be a Borel set of subgroups of $S_{\infty}$ that is closed under conjugation.

Suppose that for each $G \in \mathcal{V}$ we have a countably infinite set $\mathcal{N}_{G}$ of open subgroups of $G$ that forms a neighbourhood basis of 1 . Suppose further that the relation

$$
\mathcal{T}=\left\{(G, U): G \in \mathcal{V} \text { and } U \in \mathcal{N}_{G}\right\}
$$

is Borel, as well as isomorphism invariant in the sense that

$$
\phi: G \cong H \text { implies } V \in \mathcal{N}_{G} \leftrightarrow \phi(V) \in \mathcal{N}_{H} .
$$

Then:

(i) The isomorphism relation on $\mathcal{V}$ is Borel reducible to conjugacy of closed subgroups of $S_{\infty}$. (Moreover, a conjugating permutation can be obtained in a Borel way from an isomorphism, and vice versa.)

(ii) In particular, the isomorphism relation on $\mathcal{V}$ is classifiable by countable structures.

Proof. We assign in a Borel way to each $G \in \mathcal{V}$ a closed subgroup $\widehat{G}$ of $S_{\infty}$ so that $G \cong H$ iff $\widehat{G}$ is conjugate to $\widehat{H}$. Moreover, we ensure that $G \cong \widehat{G}$.

For $G \in \mathcal{V}$, let $\mathcal{L}(G)$ denote the set of left cosets of the subgroups in $\mathcal{N}_{G}$. The relation

$$
\mathcal{S}=\left\{((G, B), U): G \in \mathcal{V}, U \in \mathcal{N}_{G} \text { and } \exists i B=p_{i}^{G} U\right\}
$$

is Borel and has countable sections on the second component because each $\mathcal{N}_{G}$ is countable. By a result of Lusin-Novikov, in the version of $[6,18.10]$, the projection of $\mathcal{S}$ onto the first component is Borel. This projection equals $\{(G, B): G \in \mathcal{V} \wedge B \in \mathcal{L}(G)\}$. Note also that $\mathcal{L}(G)$ is countably infinite. So by the same result $[6,18.10]$ there is a Borel function $F: \mathcal{V} \rightarrow \mathcal{F}\left(S_{\infty}\right)^{\omega}$ taking a group $G \in \mathcal{V}$ to a bijection $\eta_{G}: \omega \rightarrow \mathcal{L}(G)$.

The group $G$ acts by left translation on $\mathcal{L}(G)$. So via the bijection $\eta_{G}$, the left action of an element $g$ on $\mathcal{L}(G)$ corresponds to a permutation $\Theta_{G}(g) \in$ $S_{\infty}$. We let $\widehat{G}$ be the range of $\Theta_{G}$ and verify that the map $G \rightarrow \widehat{G}$ is Borel and has the desired property. We will omit the subscript $G$ for now and simply write $\Theta$.

Claim 3.2. The map $\Theta$ is a topological group isomorphism $G \cong \Theta(G)$.

Clearly $\Theta$ is a homomorphism of groups. To show that $\Theta$ is 1-1 suppose that $g \neq 1$ and pick $V \in \mathcal{N}_{G}$ such that $g \notin V$. Then $g V \neq V$, hence $\Theta(g)\left(\eta_{G}^{-1}(V)\right) \neq \eta_{G}^{-1}(V)$, so that $\Theta(g) \neq 1$.

For continuity of $\Theta^{-1}$ at 1 , suppose $U \in \mathcal{N}_{G}$, and let $n=\eta_{G}(U)$. If $\Theta(g)(n)=n$ then $g \in U$.

For continuity of $\Theta$ at 1 , consider $C \in \mathcal{L}(G)$ and let $n=\eta_{G}(C)$. If $g C=C$ then $\Theta(g)(n)=n$. So it suffices to find a neighbourhood $W$ of 1 in $G$ such that $g \in W \rightarrow g C=C$. Choose $r \in G$ and $U \in \mathcal{N}_{G}$ such that $C=r U$. 
The desired neighbourhood $W$ is $\left\{g: r^{-1} g r \in U\right\}=r U r^{-1}$. This shows the claim.

Since $\Theta(G)$ is Polish it is a $G_{\delta}$ subgroup of $S_{\infty}$, and hence closed by the Baire category theorem $[2,1.2 .1]$.

Claim 3.3. The map $L: \mathcal{V} \rightarrow \mathcal{F}\left(S_{\infty}\right)$ sending $G$ to $\widehat{G}$ is Borel.

We use that the action by left translation $G \curvearrowright \mathcal{F}\left(S_{\infty}\right)$ is Borel, and the assignment $G \rightarrow \eta_{G}$ is Borel. In the notation of Section 2, we have to show that the preimage of $\mathcal{L}_{[\sigma]}$ under $L$ is Borel for each tuple $\sigma$ without repetitions. This preimage equals $\{G: \widehat{G} \cap[\sigma] \neq \emptyset\}$, which in turn equals $\left\{G: \exists g \in G \Theta_{G}(g) \in[\sigma]\right\}$. For each $G$ the set of such $g$ is open in $G$ because $\Theta_{G}$ is continuous. So if this set is nonempty it contains $p_{i}^{G}$ for some $i \in \omega$, where $p_{i}^{G}$ is given by Lemma 2.1. To say that $\Theta_{G}\left(p_{i}^{G}\right) \in[\sigma]$ means that

$$
\forall n, k\left[\sigma(n)=k \rightarrow p_{i}^{G} \eta_{G}(n)=\eta_{G}(k)\right\},
$$

which is a Borel property of $G$. The preimage of $\mathcal{L}_{[\sigma]}$ under $L$ equals the union of these sets over all $i$, which is therefore Borel. This shows the claim.

Suppose now that $G, H \in \mathcal{V}$. First let $\phi: G \rightarrow H$ be a topological group isomorphism. By our hypothesis that the relation $\mathcal{R}$ is isomorphism invariant, $\phi$ induces a bijection $\Phi: \mathcal{L}(G) \rightarrow \mathcal{L}(H)$ via $\Phi(r U)=\phi(r) \phi(U)$ for $U \in \mathcal{N}_{G}$. Then $\Theta_{G}(G)$ is conjugate to $\Theta_{H}(H)$ via the permutation $\alpha=\eta_{H}^{-1} \circ \Phi \circ \eta_{G}$, because for $p=\Theta_{G}(g)$ we have $\alpha \circ p \circ \alpha^{-1}=\Theta_{H}(\phi(g))$.

Next suppose that $\Theta_{G}(G)^{\alpha}=\Theta_{H}(H)$ for $\alpha \in S_{\infty}$. Then $\phi=\Theta_{H}^{-1} \circ \alpha \circ \Theta_{G}$ is a topological group isomorphism of $G$ and $H$.

Note that one can obtain $\alpha$ from $\phi$ in a Borel way, and vice versa. This shows (i).

For (ii), recall that any $S_{\infty}$-orbit equivalence relation is classifiable by countable structures by a result of Becker and Kechris [2, Thm. 2.7.3]; also see $[4$, Thm. 3.6.1].

We now apply the criterion given by the foregoing theorem to various classes of groups. We have already mentioned how to obtain the case of locally compact groups.

A closed subgroup $G$ of $S_{\infty}$ is called oligomorphic if for each $n$ there are only finitely many $n$-orbits for the natural action of $G$ on $\omega$. Clearly this property is Borel. Each open subgroup of $G$ contains an open subgroup $\left[\tau_{n}\right] \cap G$ for some $n$, where $\tau_{n}$ denotes the identity tuple $(0,1, \ldots, n)$. Since $\left[\tau_{n}\right] \cap G$ has only finitely many cosets in $G$, there are only countably many open subgroups.

A topological group $G$ is called Roelcke precompact if for each neighbourhood $U$ of 1 , there is a finite set $F$ such that $U F U=G$. If $G$ is a closed subgroup of $S_{\infty}$, we may assume that $U$ is an open subgroup of $G$, in which case the defining condition states that the double coset equivalence relation $\sim_{U}$ given by $x \sim_{U} y \leftrightarrow U x U=U y U$ has only finitely many equivalence classes.

Roelcke pre-compactness is a Borel property of subgroups of $S_{\infty}$ : given an open subgroup $U$, if $F$ as above exists we can choose the elements of $F$ among the dense sequence $p_{i}^{G}$ obtained in a Borel way as in Lemma 2.1. It suffices to require that $F$ exists for each $U=\left[\tau_{n}\right] \cap G$. 
We note that a Roelcke precompact non-Archimedean group $G$ has only countably many open subgroups: if $U$ is an open subgroup, then each $\sim_{U}$ class is a finite union of $\sim_{\left[\tau_{n}\right] \cap G}$ classes for an appropriate $n$. Now $U$ itself is the equivalence class of $1_{G}$ under $\sim_{U}$. So there are only countably many options for $U$.

Corollary 3.4. Let $\mathcal{V}$ be a Borel set of subgroups of $S_{\infty}$ that is closed under conjugation. Suppose that every group in $\mathcal{V}$ has only countably many open subgroups. Then the isomorphism problem for $\mathcal{V}$ is classifiable by countable structures.

In particular, this is the case for the oligomorphic and Roelcke precompact groups.

Proof. Let $\mathcal{N}_{G}$ be the set of all open subgroups of $G \in \mathcal{V}$. The relation $\mathcal{T}$ in Thm. 3.1 is Borel because a closed group $H \leq G$ is open in $G$ iff $G \cap\left[\tau_{n}\right] \subseteq H$ for some $n$, where as before $\tau_{n}$ is the identity tuple of length $n+1$. Next, $G \cap\left[\tau_{n}\right] \subseteq H$ is equivalent to $\forall \rho \succeq \tau_{n}([\rho] \cap G \neq \emptyset \rightarrow[\rho] \cap H \neq \emptyset)$. $\mathcal{T}$ is clearly isomorphism invariant, so all the hypotheses of the theorem hold.

3.2. Remarks. Roelcke precompactness generalises both compactness and being oligomorphic. For the second statement, note that by Tsankov [11], $G$ is Roelcke precompact iff $G$ is the inverse limit of a diagram

$$
\ldots \rightarrow G_{3} \rightarrow G_{2} \rightarrow G_{1}
$$

where each $G_{i}$ is an oligomorphic permutation group on some countable set. As pointed out in [11, before Prop. 2.2] the only Roelcke precompact and locally compact Polish groups $G$ are the compact ones.

We note that besides the Roelcke precompact groups, there are further non-Archimedean groups with only countably many open subgroups, for instance many (discrete) countable groups. For an uncountable example, consider the locally compact group $\operatorname{PSL}_{2}\left(\mathbb{Q}_{p}\right)$ : by a result of Tits, each open subgroup is either compact, or the whole group. We thank Pierre-Emmanuel Caprace for pointing out this example.

3.3. A direct construction of structures for Theorem 3.1(ii). To prove Theorem 3.1(ii) we used that any $S_{\infty}$-orbit equivalence relation $E$ is classifiable by countable structures [2, Thm. 2.7.3]. This result actually gives an $S_{\infty}$ reduction of $E$ to the logic action for an infinitary language. On the downside, the proof is quite indirect, making use of the fact that the action by left translation of $S_{\infty}$ on $\mathcal{F}\left(S_{\infty}\right)^{\omega}$ is universal, and then encoding sequences of closed sets by a countable structure based on the sequence of corresponding trees.

We now give a direct construction of the structures in Theorem 3.1(ii). Recall that the structures for a fixed countable relational language $L=$ $\left(R_{i}\right)_{i \in I}$ form a Polish space $X_{L}=\prod_{I} \mathcal{P}\left(\omega^{n_{i}}\right)$ (where $n_{i}$ is the arity of $R_{i}$ ). We now define a finite relational language $L$ and a Borel function that assigns to $G \in \mathcal{V}$ a countable $L$-structure $M_{G}$ in a such a way that groups $G, H \in \mathcal{V}$ are topologically isomorphic iff $M_{G} \cong M_{H}$ as $L$-structures.

Given a group $G$, the universe of the structure $M_{G}$ consists of the left and right cosets of subgroups in $\mathcal{N}_{G}$. Since $\mathcal{N}_{G}$ is countable and each group in $\mathcal{N}_{G}$ is open in $G$, this is a countable set. The language $L$ consists of 
two unary relations $\mathcal{L}$ and $\mathcal{R}$, denoting the left and right cosets respectively. Again by the result of Lusin-Novikov as in $[6,18.10]$ there is a Borel function taking a group $G \in \mathcal{V}$ to a bijection $\nu_{G}: \omega \rightarrow \operatorname{dom}\left(M_{G}\right)$, in such a way that even numbers go to left cosets and odd numbers to right cosets. So we may identify the elements of $M_{G}$ with natural numbers.

Furthermore, the language $L$ contains the following relations

- inclusion between cosets (of any kind),

- the ternary relation $A B \subseteq C$,

- the binary relation that $A$ is a coset of a subgroup $U$ (of the kind corresponding to $A$ ).

Note that being a subgroup is definable in $M_{G}$ because a coset $A$ is a subgroup of $G$ if and only if $A A \subseteq A$.

Claim 3.5. The function $G \mapsto M_{G}$ (for $\left.G \in \mathcal{V}\right)$ is Borel.

Proof. Given a relation symbol $R$ of arity $n$ and an $n$-tuple of numbers $\bar{a}$, we need to show that the preimage of the set of structures that satisfy $R \bar{a}$, that is, $\left\{G: M_{G} \models R \bar{a}\right\}$, is Borel in $\mathcal{F}\left(S_{\infty}\right)$.

As observed above, inclusion is a Borel relation on $\mathcal{F}\left(S_{\infty}\right)$. The preimage of the structures where the coset denoted $a_{0}$ is contained in the coset denoted $a_{1}$ is $\left\{G \in \mathcal{V}: \nu_{G}\left(a_{0}\right) \subseteq \nu_{G}\left(a_{1}\right)\right\}$. Since the assignment $G \mapsto \nu_{G}$ is Borel, this preimage is Borel. For the relation on numbers corresponding to the ternary relation $A B \subseteq C$ we argue in a similar way, using Lemma 2.4.

For the binary relation expressing that $A$ is a coset of the subgroup $U$, we first recall from Section 2 that being a subgroup is a Borel property of closed sets. Second, an even number $a$ denotes a coset of the subgroup denoted by $u$ iff $\exists i p_{i}^{G} \nu_{G}(u)=\nu_{G}(a)$, where $p_{i}^{G}$ is as in Lemma 2.1. The set of such groups $G$ is Borel. For right cosets the argument is analogous.

First suppose that $G \cong H$ via $\theta$. By the hypothesis of isomorphism invariance of the relation $\mathcal{T}$ defined in Theorem 3.1, $M_{G} \cong M_{H}$ via $\nu_{H}^{-1}$ 。 $\Theta \circ \nu_{G}$, where $\Theta$ is the map on left and on right cosets induced by $\theta$.

Next we show that $M_{G} \cong M_{H}$ implies that $G \cong H$. To $g \in G$ we associate the pair $L_{g}, R_{g}$, where $L_{g}$ is the set of left cosets in $M_{G}$ containing $g$, and $R_{g}$ the set of right cosets containing $g$. Both sets are neighbourhood bases of $g$ consisting of open sets. So we have the following properties of $L=L_{g}$ and $R=R_{g}$ :

(1) $L$ and $R$ are downward directed under inclusion.

(2) For each $A \in L$ and $B \in R$ there is $C \in L$ such that $C \subseteq A \cap B$.

(3) Each group $U \in \mathcal{N}_{G}$ has a left coset in $L$ and a right coset in $R$ (these cosets are necessarily unique).

Suppose now we have a pair $L, R$ with the properties above. We construct an element $g$ of $G$ such that $L=L_{g}$ and $R=R_{g}$.

Let $U_{0} \in \mathcal{N}_{G}$. Using that $\mathcal{N}_{G}$ is a neighbourhood basis, for each $n>0$, let $U_{n} \in \mathcal{N}_{G}$ be such that $U_{n} \leq\left[\tau_{n}\right] \cap U_{n-1}$, where as above $\tau_{n}$ is the identity tuple of length $n+1$. Define $g(n)=r_{n}(n)$ where $r_{n} \in G$ and $r_{n} U_{n}$ is the left coset of $U_{n}$ in $L$. Also define $g^{*}(n)=s_{n}^{-1}(n)$ where $U_{n} s_{n}$ is the right coset of $U_{n}$ in $R$. 
Clearly the $r_{n} \uparrow_{n+1}(n \in \omega)$ are compatible tuples with union $g$. Similarly the $\left.s_{n}^{-1}\right|_{n+1}$ are compatible tuples with union $g^{*}$. However, so far we don't know that the injective function $g$ is a permutation.

Claim 3.6. $g^{*}$ is the inverse of $g$. In particular, $g \in S_{\infty}$.

Proof. Note that $r_{n}$ determines the first $n+1$ values of any permutation $f$ in $r_{n} U_{n}$, namely $f(i)=r_{n}(i)$ for each $i \leq n$. Likewise, $h \in U_{n} s_{n}$ implies that $h^{-1} \in s_{n}^{-1} U_{n}$ and hence $h^{-1}(i)=s_{n}^{-1}(i)$ for each $i \leq n$.

Assume for a contradiction that, say $g(x)=y$ and $g^{*}(y) \neq x$. Let $n=$ $\max (x, y)$. Then $r_{n} U_{n} \cap U_{n} s_{n}=\emptyset$, contrary to (2) above: if $f \in r_{n} U_{n} \cap U_{n} s_{n}$ then by the compatibility and the observation above, $f(x)=r_{n}(x)=y$, while $f^{-1}(y)=s_{n}^{-1}(y) \neq x$.

Claim 3.7. (i) $g \in G$. (ii) $L_{g}=L$ and $R_{g}=R$.

Proof. (i) follows because $g \uparrow_{n+1}=r_{n} \uparrow_{n+1}$ and $G$ is closed. (ii) is clear from the definition of $g$ and $g^{*}=g^{-1}$.

Claim 3.8. For $a, b, c \in G$,

$a \circ b=c \leftrightarrow \forall C \in L_{c} \exists A \in L_{a} \exists B \in L_{b}[A B \subseteq C]$.

Proof. The implication from left to right holds by continuity of composition in $G$. For the converse implication, suppose that $(a \circ b)(n) \neq c(n)$. Let $C \in L_{c}$ be the left coset of $U_{n}$. Then $d(n)=c(n)$ for any $d \in C$. If $A \in L_{a}$ and $B \in L_{b}$ then $a \circ b \in A B$ so that $A B \nsubseteq \subset C$.

Now suppose that $M_{G} \cong M_{H}$ via $\rho$. Given $g \in G$, note that the pair $\rho\left(L_{g}\right), \rho\left(R_{g}\right)$ has the properties (1-3) listed above. So let $\theta(g)$ be the element of $H$ obtained from this pair. Similarly, the inverse of $\theta$ is determined by the inverse of $\rho$.

By Claim 3.6, $\theta$ preserves the composition operation. The identity of $G$ is the only element $g \in G$ such that $L_{g}=\mathcal{N}_{G}$. Since $\rho$ is an isomorphism of the structures, $\theta\left(1_{G}\right)=1_{H}$, and $\theta$ and $\theta^{-1}$ are continuous at 1 . So $\theta$ is a topological isomorphism.

\section{HARDNESS RESULT FOR ISOMORPHISM OF PROFINITE GROUPS}

Graph isomorphism is complete for $S_{\infty}$-orbit equivalence relations. We now consider the converse problem of Borel reducing graph isomorphism to isomorphism on a Borel class of nonarchimedean groups. We first consider the case of discrete groups. Essentially by a result of Mekler [7, Section 2] discussed in more detail below, graph isomorphism is Borel reducible to isomorphism of countable groups. Given a discrete group $G$ with domain $\omega$, the left translation action of $G$ on itself induces a topological isomorphism of $G$ with a discrete subgroup of $S_{\infty}$. Hence graph isomorphism is Borel reducible to isomorphism of discrete, and hence of locally compact, subgroups of $S_{\infty}$.

We now show a similar hardness result for the compact non-archimedean groups (equivalently, the separable profinite groups). Recall that a group $G$ is step 2 nilpotent (nilpotent-2 for short) if it satisfies the law $[[x, y], z]=1$. 
Equivalently, the commutator subgroup is contained in the center. For a prime $p$, the group of unitriangular matrices

$$
\mathrm{UT}_{3}^{3}(\mathbb{Z} / p \mathbb{Z})=\left\{\left(\begin{array}{lll}
1 & a & c \\
0 & 1 & b \\
0 & 0 & 1
\end{array}\right): a, b, c \in \mathbb{Z} / p \mathbb{Z}\right\}
$$

is an example of a nilpotent-2 group of exponent $p$.

4.1. Completion. We need some preliminaries on the completion of a group $G$ with respect to a system of subgroups of finite index. We follow $[9$, Section 3.2]. Let $\mathcal{V}$ be a set of normal subgroups of finite index in $G$ such that $U, V \in \mathcal{V}$ implies that there is $W \in \mathcal{V}$ with $W \subseteq U \cap V$. We can turn $G$ into a topological group by declaring $\mathcal{V}$ a basis of neighbourhoods of the identity. In other words, $M \subseteq G$ is open if for each $x \in M$ there is $U \in \mathcal{V}$ such that $x U \subseteq M$.

The completion of $G$ with respect to $\mathcal{V}$ is the inverse limit

$$
G_{\mathcal{V}}=\lim _{U \in \mathcal{V}} G / U
$$

where $\mathcal{V}$ is ordered under inclusion and the inverse system is equipped with the natural maps: for $U \subseteq V$, the map $p_{U, V}: G / U \rightarrow G / V$ is given by $g U \mapsto g V$. The inverse limit can be seen as a closed subgroup of the direct product $\prod_{U \in \mathcal{V}} G / U$ (where each group $G / U$ carries the discrete topology), consisting of the functions $\alpha$ such that $p_{U, V}(\alpha(g U))=g V$ for each $g$. Note that the map $g \mapsto(g U)_{U \in \mathcal{V}}$ is a continuous homomorphism $G \rightarrow G_{\mathcal{V}}$ with dense image; it is injective iff $\bigcap \mathcal{V}=\{1\}$.

If the set $\mathcal{V}$ is understood from the context, we will usually write $\widehat{G}$ instead of $G_{\mathcal{V}}$.

4.2. Review of Mekler's construction. Fix an odd prime $p$. The main construction in Mekler [7, Section 2] associates to each symmetric and irreflexive graph $A$ a nilpotent-2 exponent-p group $G(A)$ in such a way that isomorphic graphs yield isomorphic groups. In the countable case, the map $G$ is Borel when viewed as a map from the Polish space of countable graphs to the space of countable groups.

To recover $A$ from $G(A)$, Mekler uses a technical restriction on the given graphs.

Definition 4.1. A symmetric and irreflexive graph is called nice if it has no triangles, no squares, and for each pair of distinct vertices $x, y$, there is a vertex $z$ joined to $x$ and not to $y$.

Mekler [7] proves that a nice graph $A$ can be interpreted in $G(A)$ using first-order formulas without parameters. (See [5, Ch. 5] for background on interpretations.) In particular, for nice graphs $A, B$ we have $A \cong B$ iff $G(A) \cong G(B)$. Since isomorphism of nice graphs is Borel complete for $S_{\infty}$-orbit equivalence relations, so is isomorphism of countable nilpotent-2 exponent $p$ groups. For an alternative write-up of Mekler's construction see $[5$, A.3].

In the following all graphs will be symmetric, irreflexive, and have domain $\omega$. Such a graph is thus given by its set of edges $A \subseteq\{(r, s): r<s\}$. We write $r A s$ (or simply $r s$ if $A$ is understood) for $(r, s) \in A$. 
Let $F$ be the free nilpotent-2 exponent- $p$ group on free generators $x_{0}, x_{1}, \ldots$ For $r \neq s$ we write

$$
x_{r, s}=\left[x_{r}, x_{s}\right] .
$$

As noted in [7], the centre $Z(F)$ of $F$ is an elementary abelian $p$-group (so an $\mathbb{F}_{p}$ vector space) with basis $x_{r, s}$ for $r<s$. Given a graph $A$, Mekler sets

$$
G(A)=F /\left\langle x_{r, s}: r A s\right\rangle .
$$

In particular $F=G(\emptyset)$. The centre $Z=Z(G(A))$ is an abelian group of exponent $p$ freely generated by the $x_{r, s}$ such that $\neg r A s$. Also $G(A) / Z$ is an abelian group of exponent $p$ freely generated by the $Z x_{i}$. (Intuitively, when defining $G(A)$ as a quotient of $F$, exactly the commutators $x_{r, s}$ such that $r A s$ are deleted. We make sure that no vertices are deleted.)

Lemma 4.2 (Normal form for $G(A),[7,5]$ ). Every element $c$ of $Z$ can be uniquely written in the form $\prod_{(r, s) \in L} x_{r, s}^{\beta_{r, s}}$ where $L \subseteq \omega \times \omega$ is a finite set of pairs $(r, s\rangle$ with $r<s$ and $\neg r A s$, and $0<\beta_{r, s}<p$.

Every element of $G(A)$ can be uniquely written in the form $c \cdot v$ where $c \in Z$, and $v=\prod_{i \in D} x_{i}^{\alpha_{i}}$, for $D \subseteq \omega$ finite and $0<\alpha_{i}<p$. (The product $\prod_{i \in D} x_{i}^{\alpha_{i}}$ is interpreted along the indices in ascending order.)

4.3. Hardness result for isomorphism of profinite groups. The following first appeared in preprint form in [8].

Theorem 4.3. Let $p \geq 3$ be a prime. Any $S_{\infty}$ orbit equivalence relation can be Borel reduced to isomorphism between profinite nilpotent-2 groups of exponent $p$.

We note that isomorphism on the class of abelian compact subgroups of $S_{\infty}$ is not Borel-above graph isomorphism as shown in [8]. So in a sense the class of nilpotent-2 groups of fixed exponent $p$ is the smallest possible.

Proof. The proof is based on Mekler's, replacing the groups $G(A)$ he defined by their completions $\widehat{G}(A)$ with respect to a suitable basis of neighbourhoods of the identity. Given a graph $A$, let $R_{n}$ be the normal subgroup of $G(A)$ generated by the $x_{i}, i \geq n$. Note that $G(A) / R_{n}$ is a finitely generated nilpotent torsion group, and hence finite. Let $\widehat{G}(A)$ be the completion of $G(A)$ with respect to the set $\mathcal{V}=\left\{R_{n}: n \in \omega\right\}$ (see Subsection 4.1). By Lemma 4.2 we have $\bigcap_{n} R_{n}=\{1\}$, so $G(A)$ embeds into $\widehat{G}(A)$.

In set theory one inductively defines $0=\emptyset$ and $n=\{0, \ldots, n-1\}$ to obtain the natural numbers; this will save on notation here. A set of coset representatives for $G(A) / R_{n}$ is given by the $c \cdot v$ as in Lemma 4.2, where $D \subseteq n$ and $E \subseteq n \times n$. The completion $\widehat{G}(A)$ of $G(A)$ with respect to the $R_{n}$ consists of the maps $\rho \in \prod_{n} G(A) / R_{n}$ such that $\rho\left(g R_{n+1}\right)=g R_{n}$ for each $n \in \omega$ and $g \in G(A)$.

If $\rho\left(g R_{n+1}\right)=h R_{n}$ where $h=c \cdot v$ is a coset representative, then we can define a coset representative $c^{\prime} \cdot v^{\prime}$ for $g R_{n+1}$ as follows: we obtain $c^{\prime}$ from $c$ by potentially appending to $c$ factors involving the $x_{r, n}$ for $r<n$, and $v^{\prime}$ from $v$ by potentially appending a factor $x_{n}^{\alpha_{n}}$. So we can view $\rho$ as given by multiplying two formal infinite products: 
Lemma 4.4 (Normal form for $\widehat{G}(A))$. Every $c \in Z(\widehat{G}(A))$ can be written uniquely in the form $\prod_{(r, s) \in L} x_{r, s}^{\beta_{r, s}}$ where $L \subseteq \omega \times \omega$ is a set of pairs $(r, s\rangle$ with $r<s, \neg r A s$, and $0<\beta_{r, s}<p$.

Every element of $\widehat{G}(A)$ can be written uniquely in the form $c \cdot v$, where $v=\prod_{i \in D} x_{i}^{\alpha_{i}}, c \in Z(\widehat{G}(A)), D \subseteq \omega$, and $0<\alpha_{i}<p$ (the product is taken along ascending indices).

We can define the infinite products above explicitly as limits in $\widehat{G}(A)$. We view $G(A)$ as embedded into $\widehat{G}(A)$. Given formal products as above, let

$$
v_{m}=\prod_{i \in D \cap m} x_{i}^{\alpha_{i}} \text { and } c_{m}=\prod_{(r, s) \in L \cap m \times m} x_{r, s}^{\beta_{r s}} .
$$

For $k \geq n$ we have $v_{k}^{-1} v_{n} \in R_{n}$ and $c_{k}^{-1} c_{n} \in R_{n}$. So $v=\lim _{n} v_{n}$ and $c=\lim _{n} c_{n}$ exist in $\widehat{G}(A)$ and equal the values of the formal products as defined above.

Each nilpotent-2 group satisfies the distributive law $[x, y z]=[x, y][x, z]$. This implies that $\left[x_{r}^{\alpha}, x_{s}^{\beta}\right]=x_{r, s}^{\alpha \beta}$. The following lemma generalises to infinite products the expression for commutators that were obtained using these identities in [7, p. 784] (and also in [5, proof of Lemma A.3.4]).

Lemma 4.5 (Commutators). Let $D, E \subseteq \omega$. The following holds in $\widehat{G}(A)$.

$$
\left[\prod_{r \in D} x_{r}{ }^{\alpha_{r}}, \prod_{s \in E} x_{s}^{\beta_{s}}\right]=\prod_{r \in D, s \in E, r<s, \neg r s} x_{r, s}^{\alpha_{r} \beta_{s}-\alpha_{s} \beta_{r}}
$$

Proof. Based on the case of finite products, by continuity of the commutator operation and using the expressions for limits above, we have

$$
\begin{aligned}
{\left[\prod_{r \in D} x_{r}^{\alpha_{r}}, \prod_{s \in E} x_{s}^{\beta_{s}}\right] } & =\left[\lim _{n} \prod_{r \in D \cap n} x_{r}^{\alpha_{r}}, \lim _{n} \prod_{s \in E \cap n} x_{s}^{\beta_{s}}\right] \\
& =\lim _{n} \prod_{r \in D, s \in E, r<s<n, \neg r s} x_{r, s}^{\alpha_{r} \beta_{s}-\alpha_{s} \beta_{r}} \\
& =\prod_{r \in D, s \in E, r<s, \neg r s} x_{r, s}^{\alpha_{r} \beta_{s}-\alpha_{s} \beta_{r}} .
\end{aligned}
$$

Let $C(g)$ denote the centraliser of a group element $g$. The following is a direct consequence of Lemma 4.5.

Lemma 4.6. Let $w \in \widehat{G}(A)$. If $0<\gamma<p$ we have $C\left(w^{\gamma}\right)=C(w)$.

Mekler's argument employs the niceness of $A$ to show that a copy of the set of vertices of the given graph is first-order definable in $G(A)$. The copy of vertex $i$ is a certain definable equivalence class of the generator $x_{i}$. He provides a first-order interpretation $\Gamma$ without parameters such that $\Gamma(G(A)) \cong A$. We will show that his interpretation has the same effect in the profinite case: $\Gamma(\widehat{G}(A)) \cong A$.

We first summarize Mekler's interpretation $\Gamma$. Let $H$ be a group with centre $Z(H)$.

- For $a \in H$ let $\bar{a}$ denote the coset $a Z(H)$. 
- Write $\bar{a} \sim \bar{b}$ if $C(a)=C(b)$. Let $[\bar{a}]$ be the $\sim$ equivalence class of $\bar{a}$. (Thus, for $c \in Z(H)$ we have $[\bar{c}]=\{Z(H)\}$.)

Given a group $H$ we define the vertex set of the graph $\Gamma(H)$ to be

$$
\Gamma(H)=\{[\bar{a}]: a \in H \backslash Z(H) \text { and }|[\bar{a}]|=p-1 \text { and }
$$

$\exists w \in H \backslash Z(H)$ such that $[\bar{w}] \neq[\bar{a}]$ and $[a, w]=1\}$

and the edge relation $R$ is given by

$$
[\bar{a}] R[\bar{b}] \text { if }[\bar{a}] \neq[\bar{b}] \text { and }[a, b]=1 \text {. }
$$

Note that the vertex set is interpretable and the edge relation definable. We are ready to verify that a nice graph can be recovered from its associated profinite group via the interpretation $\Gamma$.

Lemma 4.7. For a nice graph $A$, we have $\Gamma_{2}(\widehat{G}(A)) \cong A$ via $\left[\overline{x_{i}}\right] \mapsto i$.

We follow the outline of the proof of [7, Lemma 2.2]. The notation there will be adapted to $\widehat{G}(A)$ via allowing infinite products.

Convention 4.8. Henceforth, in products of the form $\prod_{i \in D} x_{i}^{\alpha_{i}}$ we will assume that $D \subseteq \omega$ is nonempty and $0<\alpha_{i}<p$ for each $i \in D$. We also set $\alpha_{i}=0$ for $i \notin D$. Expressions and calculations involving number exponents $\alpha$ will all be modulo p; e.g. $\alpha \neq 0$ means that $\alpha \not \equiv 0 \bmod p$.

Clearly the edge relation is the only possible one, so it suffices to show that

$$
\Gamma(\widehat{G}(A))=\left\{\left[\bar{x}_{i}\right]: i \in \omega\right\} .
$$

Now let $v \notin Z(\widehat{G}(A))$. We may assume that $v=\prod_{i \in D} x_{i}{ }^{\alpha_{i}}$ satisfying the conventions above. We will show that

$$
[\bar{v}]=\left[\bar{x}_{e}\right] \text { for some } e \text { if and only if }|[\bar{v}]|=p-1 \text { and } \exists \bar{w}[\bar{v}] R[\bar{w}] .
$$

We distinguish four cases: in Case 1 we check that $v$ satisfies the two conditions on the right hand side, in Case 2-4 that it fails. Recall that $r s$ is short for $r A s$, i.e. that vertices $r, s$ are joined.

Case 1: $D=\{r\}$ for some $r$.

Note that we have $\bar{v}=\bar{x}_{r}^{\alpha}$ for some $\alpha \neq 0$. Suppose $\bar{u} \sim \bar{v}$ for $u=$ $\prod_{k \in E} x_{k}{ }^{\beta_{k}}$. Then $E=\{r\}$. For, if $k \in E, k \neq r$, then by niceness of $A$ there is an $s$ such that $r s \wedge \neg k s$, so that $x_{s} \in C(v) \backslash C(u)$ by Lemma 4.5. Thus $\bar{u}=\bar{x}_{r}^{\beta}$ with $0<\beta<p$ and hence $|[\bar{v}]|=p-1$.

For the second condition, by niceness of $A$ we pick $i \neq r$ such that $i r$, and let $\bar{w}=\bar{x}_{i}$.

Case 2: $D=\{r, s\}$ for some $r, s$ such that $r A s$ (and hence $r \neq s$ ).

We show that $[\bar{v}]=\left\{\bar{x}_{r}^{\alpha} \bar{x}_{s}^{\beta}: 0<\alpha, \beta<p\right\}$, and hence this equivalence class has size $(p-1)^{2}$. To this end we verify:

Claim. Let $w=\prod_{k \in E} x_{k}{ }^{\beta_{k}}$. Let $\alpha, \beta \neq 0$. We have

$$
\left[w, \bar{x}_{r}^{\alpha} \bar{x}_{s}^{\beta}\right]=1 \leftrightarrow E \subseteq\{r, s\} .
$$


For the implication from left to right, if there is $k \in E \backslash\{r, s\}$, then $k r$ and $k s$, so $A$ has a triangle. Hence $E \subseteq\{r, s\}$. The converse implication follows by distributivity and since $\left[x_{r}, x_{s}\right]=1$. This shows the claim.

By the claim, $C\left(\bar{x}_{r}^{\alpha} \bar{x}_{s}^{\beta}\right)=C(v)$ for each $\alpha, \beta \neq 0$. On the other hand, if $K \subset\{r, s\}$ then $C\left(\prod_{i \in K} x_{i}^{\beta_{i}}\right) \nsubseteq \subseteq C(v)$ by niceness, and if $K \nsubseteq\{r, s\}$ then $v \notin C\left(\prod_{i \in K} x_{i}^{\beta_{i}}\right)$ by the claim again.

Case 3: Neither Case 1 nor 2, and there is an $\ell$ such that $i \ell$ for each $i \in D$. Claim. $[\bar{v}]=\left\{\bar{v}^{\gamma} \bar{x}_{\ell}^{\beta}: 0<\gamma<p \wedge 0 \leq \beta<p\right\}$, and hence $|[\bar{v}]|=p(p-1)$.

First an observation: suppose that $\left[v^{\gamma} x_{\ell}^{\beta}, w\right]=1$ where $\gamma, \beta$ are as above and $w=\prod_{k \in E} x_{k}{ }^{\beta_{k}}$. Then $E \subseteq D \cup\{\ell\}$. For, since Cases 1 and 2 don't apply, there are distinct $p, q \in D \backslash\{\ell\}$. Since $A$ has no squares, $\ell$ is the only vertex adjacent to both $p$ and $q$. Hence, given $j \in E \backslash\{\ell, p, q\}$, we have $\neg p j \vee \neg q j$, say the former. Then $j \in D$, for otherwise, in Lemma 4.5, in the expansion of $\left[v^{\gamma} x_{\ell}^{\beta}, w\right]$, we get a term $x_{p, j}^{m}$ with $m \neq 0$ and $\neg p j$ (assuming that $p<j$, say).

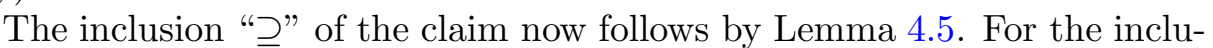
sion " $\subseteq$ " suppose that $[v, w]=1$ where $w=\prod_{k \in E} x_{k}{ }^{\beta_{k}}$. Then $E \subseteq D \cup\{\ell\}$ by our observation. By Lemma 4.5 and the case hypothesis we now have

$$
[v, w]=\prod_{r, s \in D \backslash\{\ell\}, r<s, \neg r s} x_{r, s}^{\alpha_{r} \beta_{s}-\alpha_{s} \beta_{r}} .
$$

Let $m=\min (D \backslash\{\ell\})$. Since $\alpha_{m} \neq 0$ we can pick $\gamma$ such that $\beta_{m}=\gamma \alpha_{m}$. Since $A$ has no triangles we have $\neg r s$ for any $r<s$ such that $r, s \in D \backslash\{\ell\}$, and hence $\alpha_{r} \beta_{s}=\alpha_{s} \beta_{r}$. By induction on the elements $s$ of $D \backslash\{\ell\}$ this yields $\beta_{s}=\gamma \alpha_{s}$ : if we have it for some $r<s$ in $D \backslash\{\ell\}$ then $\beta_{r} \beta_{s}=\gamma \alpha_{r} \beta_{s}=\gamma \alpha_{s} \beta_{r}$. Hence $\beta_{s}=\gamma \alpha_{s}$, for if $\beta_{r} \neq 0$ we can cancel it, and if $\beta_{r}=0$ then also $\beta_{s}=0$ because $\alpha_{r} \neq 0$.

This shows that $\bar{w}=\bar{v}^{\gamma} \bar{x}_{\ell}^{\beta}$ for some $\beta$. In particular, $C(v)=C(w)$ implies that $\bar{w}$ has the required form.

Case 4: Neither Case 1, 2 or 3.

Claim. $[\bar{v}]=\left\{\bar{v}^{\gamma}: 0<\gamma<p\right\}$, so this class has $p-1$ elements. Further, there is no $\bar{w}$ such that $[\bar{v}] R[\bar{w}]$.

The inclusion " $\supseteq$ " of the first statement follows from Lemma 4.6. We now verify the converse inclusion and the second statement. By case hypothesis there are distinct $\ell_{0}, \ell_{1} \in D$ such that $\neg \ell_{0} \ell_{1}$. Since $A$ has no squares there is at most one $q \in D$ such that $\ell_{0} q \wedge \ell_{1} q$. If $q$ exists, as we are not in Case 3 we can choose $q^{\prime} \in D$ such that $\neg q^{\prime} q$.

We define a linear order $\prec$ on $D$, which is of type $\omega$ if $D$ is infinite. It begins with $\ell_{0}, \ell_{1}$, and is followed by $q^{\prime}, q$ if they are defined. After that we proceed in ascending order for the remaining elements of $D$. Then for each $v \in D \backslash\{\min D\}$ there is $u \prec v$ in $D$ (in fact among the first three elements) such that $\neg u v$.

Suppose now that $[v, w]=1$ where $w=\prod_{k \in E} x_{k}{ }^{\beta_{k}}$. Then $E \subseteq D$ : if $s \in E \backslash D$ there is $r \in D$ such that $\neg r s$. This implies $\alpha_{r} \beta_{s}=\alpha_{s} \beta_{r}$, but $\alpha_{s}=0$ while the left hand side is $\neq 0$, contradiction.

By a slight variant of Lemma 4.5, using that $\prec$ eventually agrees with $<$, we now have $[v, w]=\prod_{r, s \in D, r \prec s, \neg r s} x_{r, s}^{\alpha_{r} \beta_{s}-\alpha_{s} \beta_{r}}$. Choose $\gamma$ such that 
$\gamma \alpha_{\ell_{0}}=\beta_{\ell_{0}}$. By induction along $(D, \prec)$ we see that $\beta_{s}=\gamma \alpha_{s}$ for each $s \in D$ : if $\ell_{0} \prec s$ choose $r \prec s$ such that $\neg r s$. Since $\alpha_{r} \beta_{s}=\alpha_{s} \beta_{r}$, as in Case 3 above we may conclude that $\beta_{s}=\gamma \alpha_{s}$.

This shows that $\bar{v}^{\gamma}=\bar{w}$. Further, if $[\bar{w}] \neq[\overline{1}]$ then $\gamma \neq 0$ so that $[\bar{v}]=[\bar{w}]$, as required.

It is important in the above argument to allow infinitely generated groups: For profinite groups that are (topologically) finitely generated, the isomorphism relation is smooth, i.e., Borel reducible to the identity relation on $\mathbb{R}$. To see this, we rely on the result that two finitely generated profinite groups are isomorphic iff they have the same finite quotients (up to isomorphism); see [3, Prop. 16.10.7]. Note that to a finitely generated profinite group, one can in a Borel way assign the set of isomorphism types of its finite quotients. For an alternative proof not relying on [3, Prop. 16.10.7] see the preprint [8, Thm. 3.1]. Also see [8] for the observation that identity on $\mathbb{R}$ is Borel reducible to isomorphism of finitely generated profinite groups.

\section{REFERENCES}

[1] G. Ahlbrandt and M. Ziegler. Quasi finitely axiomatizable totally categorical theories. Annals of Pure and Applied Logic, 30(1):63-82, 1986.

[2] H. Becker and A. Kechris. The descriptive set theory of Polish group actions, volume 232. Cambridge University Press, 1996.

[3] M. Fried and M. Jarden. Field arithmetic, volume 11. Springer Science \& Business Media, 2006.

[4] Su Gao. Invariant descriptive set theory, volume 293 of Pure and Applied Mathematics (Boca Raton). CRC Press, Boca Raton, FL, 2009.

[5] W. Hodges. Model Theory. Encyclopedia of Mathematics. Cambridge University Press, Cambridge, 1993.

[6] A. S. Kechris. Classical descriptive set theory, volume 156. Springer-Verlag New York, 1995.

[7] A. Mekler. Stability of nilpotent groups of class 2 and prime exponent. The Journal of Symbolic Logic, 46(04):781-788, 1981.

[8] A. Nies. The complexity of isomorphism between countably based profinite groups. arXiv preprint arXiv:1604.00609, 2016.

[9] L. Ribes and P. Zalesskii. Profinite groups. Springer, 2000.

[10] C. Rosendal and J. Zielinski. Compact metrizable structures and classification problems. arXiv preprint arXiv:1610.00370, 2016.

[11] T. Tsankov. Unitary representations of oligomorphic groups. Geometric and Functional Analysis, 22(2):528-555, 2012.

A. S. Kechris, Department of Mathematics, Caltech, Pasadena CA 91125, KECHRIS@CALTECH. EDU

A. Nies, Department of Computer Science, Private Bag 92019, The UniverSITY OF AUCKLAND, ANDRE@CS.AUCKLAND.AC.nZ

K. Tent, Mathematisches Institut, Einsteinstrasse 62, Universität Münster, 48149 MÜNSTER 